VOL. 63 (2001) [299-320]

\title{
AN EXTENSION OF THE GRÜNWALD-MARCINKIEWICZ INTERPOLATION THEOREM
}

\author{
T.M. Mills AND P. VÉRtesi
}

\begin{abstract}
Just over 60 years ago, G. Grünwald and J. Marcinkiewicz discovered a divergence phenomenon pertaining to Lagrange interpolation polynomials based on the Chebyshev nodes of the first kind. The main result of the present paper is an extension of their now classical theorem. In particular, we shall show that this divergence phenomenon occurs for odd higher order Hermite-Fejér interpolation polynomials of which Lagrange interpolation polynomials form one special case.
\end{abstract}

\section{The Result of GrünWald and Marcinkiewicz}

We begin by recalling the result of Grünwald and Marcinkiewicz. Let $\mathbf{Z}^{+}=$ $\{1,2,3, \ldots\}$. Consider the infinite, triangular array of points in $[-1,1]$ denoted by

$$
T:=\left(x_{k, n}=\cos ((2 k-1) \pi /(2 n)): k=1,2, \ldots, n ; n \in \mathrm{Z}^{+}\right) .
$$

For each $n$, the points $\left\{x_{1, n}, x_{2, n}, \ldots, x_{n, n}\right\}$ are the distinct zeros of the Chebyshev polynomial of the first kind $T_{n}(x):=\cos n \theta$ where $x \in[-1,1], x=\cos \theta$, and $\theta \in[0, \pi]$.

Let $f:[-1,1] \rightarrow \mathbf{R}$. Then, for each $n \in \mathbf{Z}^{+}$, define the Lagrange interpolation polynomial $L_{n-1}(T, f)(x)=L_{n-1}(T, f, x)$ to be the unique polynomial of degree $n-1$ or less such that $L_{n-1}\left(T, f, x_{k, n}\right)=f\left(x_{k, n}\right) \quad(k=1,2, \ldots, n)$. The study of $L_{n-1}(T, f, x)$ as an approximation to $f(x)$ has a long and interesting history. (See Natanson [7], Rivlin [8], Szabados and Vértesi [12].) A classical negative result discovered by Géza Grünwald [4], [5] and Józef Marcinkiewicz [6], independently of each other, is the following. (See Natanson [7] for a proof, but only for the open interval $(-1,1)$.)

Theorem 1. (G. Grünwald, J. Marcinkiewicz) There exists a function $f \in$ $C[-1,1]$ such that, for all $x \in[-1,1]$, the sequence $\left\{L_{n-1}(T, f, x): n \in Z^{+}\right\}$diverges.

Received 5th June, 2000

T.M. Mills acknowledges support provided under an exchange agreement between La Trobe University and Technical University of Budapest. P. Vértesi acknowledges support provided by a CRA/La Trobe University Distinguished Visiting Fellowship and by the Hungarian National Foundation for Scientific Research (Grants No. T7570, T17425, T22943). The authors thank Professor M.J.D. Powell and Dr Simon J. Smith for comments on an earlier draft of this paper.

Copyright Clearance Centre, Inc. Serial-fee code: 0004-9727/01 \$A2.00+0.00. 
Theorem 1 is important for two reasons.

First, it shows, dramatically, that Lagrange interpolation polynomials may be very poor approximating tools. While it is well known that, in general, Lagrange interpolation has its faults, it is widely accepted that Lagrange interpolation based on the Chebyshev nodes can be quite useful. Theorem 1 shows the limitations of Lagrange interpolation even on these nodes.

Second, it is well known that there are many similarities between the approximation properties of

(i) the partial sums of the Fourier-Chebyshev expansion of $f \in C \mid-1,1]$ and

(ii) the Lagrange interpolation polynomials $L_{n-1}(f, x)$.

These similarities can be used with great effect. For example, a result known for (i) will suggest an analogous result for (ii). However, the analogy is not perfect. A famous result of Carleson [2] implies that if $f \in C[-1,1]$ then the partial sums of the FourierChebyshev expansion of $f$ converge to $f$ almost everywhere in $[-1,1]$. On the other hand, Theorem 1 shows that this is certainly not the case for the interpolating polynomials. Thus, Theorem 1 adds to our understanding of the relationship between (i) and (ii).

The main result of this paper is a generalisation of Theorem 1. We shall show that the Grünwald-Marcinkiewicz divergence phenomenon occurs, not only for Lagrange interpolation, but for certain higher order Hermite-Fejér interpolation processes as well. In Section 2 we describe the setting for the main result and state this result. Section 3 defines some notation and Section 4 presents a sequence of technical lemmas. The proof of the main result is divided into two parts: the first part appears in Section 5 and the second part in Section 6.

\section{Higher ORDER HeRMite-FEJÉr INTERPOLATION}

In this section, we recall the basic concepts of higher order Hermite-Fejér interpolation polynomials and we state the main result of the paper.

Let $m \in \mathbf{Z}^{+}$and let $f:[-1,1] \rightarrow \mathbf{R}$. Then, for each $n \in \mathbf{Z}^{+}$, there is a unique polynomial $H_{m, n}(T, f)(x)=H_{m, n}(T, f, x)$ of degree $m n-1$, or less, such that

$$
H_{m, n}\left(T, f, x_{k, n}\right)=f\left(x_{k, n}\right) \quad(k=1,2, \ldots, n)
$$

and

$$
H_{m, n}^{(j)}\left(T, f, x_{k, n}\right)=0 \quad(k=1,2, \ldots, n ; j=1,2, \ldots, m-1)
$$

where $H_{m, n}^{(j)}(T, f, x)$ denotes the $j$-th derivative of $H_{m, n}(T, f, x)$ with respect to $x$.

We refer to $H_{m, n}(T, f, x)$ as a higher order Hermite-Fejér interpolation polynomial (the order being $m-1$ ) corresponding to the function $f$ and the $n$-th row of the matrix 
of nodes $T$. Recently there has been considerable interest in such polynomials for the following reason.

In the case $m=1$, the interpolation polynomials $H_{m, n}(T, f, x)$ are merely the Lagrange interpolation polynomials $L_{n-1}(T, f, x)$ discussed in Section 1 . In the case $m=2$, the interpolation polynomials $H_{m, n}(T, f, x)$ become the well known Hermite-Fejér interpolation polynomials which behave quite differently from Lagrange interpolation polynomials. (See Natanson [7], Rivlin [8], or Szabados and Vértesi [12].) Thus, the interpolation polynomials $H_{m, n}(T, f, x)$ generalise these classical interpolation polynomials. For an excellent survey of these polynomials see Smith [10].

What happens when $m>2$ ? It appears that, in many ways, the behaviour of $H_{m, n}(T, f, x)$ is determined by the parity of $m$.

If $m$ is odd, we expect that $H_{m, n}(T, f, x)$ may behave like $H_{1, n}(T, f, x)=L_{n-1}(T, f, x)$. For example, Szabados [11, Theorem 2] has shown that the classical theorem of G. Faber concerning the divergence of Lagrange interpolation can be extended to a theorem for odd order Hermite-Fejér interpolation polynomials.

If $m$ is even, we expect that $H_{m, n}(T, f, x)$ may behave like $H_{2, n}(T, f, x)$. For example, Vértesi [13, (1.4), (1.5), Theorem 2.3] has shown that certain convergence theorems for Hermite-Fejér interpolation polynomials can be extended to theorems for even order Hermite-Fejér interpolation polynomials.

There is no general theorem to bear out this expectation - indeed, in some specific cases, our expectations are not realised! (For example, see Byrne et al. [1].) However, this theme has been the basis for many research investigations and so too it is in this paper.

The aim of this paper is to show that Theorem 1, which is concerned with the operator $L_{n-1}=H_{1, n}$, can be extended to a result for the operator $H_{m, n}$ where $m$ is any odd number. We shall prove the following result.

Theorem 2. Let $m \in\{1,3,5,7, \ldots\}$. Then, there exists a function $f \in C[-1,1]$ such that, for all $x \in[-1,1]$,

$$
\limsup _{n \rightarrow \infty}\left|H_{m, n}(T, f, x)\right|=+\infty .
$$

\section{NOTATION}

Often we shall write $x_{k}$ for $x_{k, n}=\cos ((2 k-1) \pi /(2 n))$ and $\theta_{k}$ for $\theta_{k, n}=(2 k-1) \pi /(2 n)$ provided that no confusion arises. If $\psi \in C[-1,1]$ we define $\|\psi\|:=\sup \{|\psi(x)|:-1 \leqslant$ $x \leqslant 1\}$. We shall use $c$ or $c_{1}, c_{2}, \ldots$ to denote absolute positive constants; repeated use of the same symbol does not imply equal values of the corresponding constants.

It will be necessary to refer to the fundamental polynomials of higher order Hermite interpolation. The following technicalities and notation can be found in Szabados [11]. Suppose that $m, n$ are natural numbers and $\left\{t_{1}, t_{2}, \ldots, t_{n}\right\}$ are distinct points in $(-1,1]$. 
Then there exist unique polynomials $\left\{A_{j k n}: 0 \leqslant j \leqslant m-1,1 \leqslant k \leqslant n\right\}$ such that the degree of $A_{j k n}(x)$ is at most $m n-1$, and

$$
A_{j k n}^{(r)}\left(t_{s}\right)=\delta_{j r} \delta_{k s} \quad(j, r=0,1, \ldots, m-1 ; k, s=1,2, \ldots, n) .
$$

These polynomials are the fundamental polynomials of Hermite interpolation. Of course, the polynomial $A_{j k n}$ also depends on $m$ and on the nodes $t_{1}, t_{2}, \ldots, t_{n}$. Our notation does not make this dependence explicit, but, throughout this paper, $m$ is a fixed positive, odd number and, in any situation, the corresponding nodes will be clear.

The fundamental polynomials are pertinent to our discussion for several reasons including the fact that we can write

$$
H_{m, n}(T, f, x)=\sum_{k=1}^{n} f\left(x_{k, n}\right) A_{0 k n}(x) .
$$

We shall usually write $H_{m, n}(f, x)$ for $H_{m, n}(T, f, x)$.

\section{LEMMAS}

Our proof of Theorem 2 uses the general structure of the proof of Theorem 1 in Natanson [7] together with more recent results concerning higher order Hermite-Fejér interpolation polynomials.

Lemma 1.

(i) If $n \in Z^{+}$and $S_{n}=\left\{x_{k, n}=\cos ((2 k-1) \pi / 2 n): k=1,2, \ldots, n\right\}$ then $S_{n} \cap S_{n+1}=\emptyset$.

(ii) If $S$ is a finite subset of $(-1,1)$ then there exists arbitrarily large values of $n$ for which $S_{n} \cap S=\emptyset$ and $S_{n+1} \cap S=\emptyset$ or $\{0\}$.

Proof: See Natanson [7, p. 36].

Lemma 2. If $m \in\{1,3,5, \ldots\}$ then there exists a constant $c_{m}>0$ such that if $n \in Z^{+}$and $k \in\{1,2, \ldots, n\}$ then $\left\|A_{0 k n}\right\| \leqslant c_{m}$.

Proof: See Smith [9].

LEMma 3. Suppose that $m$ is a fixed element of $\{1,3,5,7, \ldots\}, n$ is a fixed element of $\boldsymbol{Z}^{+}$, and $\left\{t_{1}, t_{2}, \ldots, t_{n}\right\}$ are $n$ fixed, distinct nodes in $[-1,1]$. Let $\phi \in C^{m-1}[-1,1]$ be such that $\phi^{(j)}\left(t_{k}\right)=0(1 \leqslant k \leqslant n ; 1 \leqslant j \leqslant m-1)$ and $\varepsilon>0$. Then there exists a polynomial $R(x)$ such that $R\left(t_{k}\right)=\phi\left(t_{k}\right)(1 \leqslant k \leqslant n), R^{(j)}\left(t_{k}\right)=0(1 \leqslant k \leqslant n ; 1 \leqslant j \leqslant$ $m-1)$ and $\|R-\phi\| \leqslant \varepsilon$.

Proof: Let $A_{j k n}(x) \quad(1 \leqslant k \leqslant n ; 0 \leqslant j \leqslant m-1)$ be the fundamental polynomials of higher order Hermite interpolation based on the nodes $\left\{t_{1}, \ldots, t_{n}\right\}$. Let $P(x)$ be a polynomial to be specified and define the polynomial $s(x)$ by

$$
s(x):=\sum_{k=1}^{n} A_{0 k n}(x)\left(\phi\left(t_{k}\right)-P\left(t_{k}\right)\right)+\sum_{j=1}^{m-1} \sum_{k=1}^{n} A_{j k n}(x)\left(\phi^{(j)}\left(t_{k}\right)-P^{(j)}\left(t_{k}\right)\right) .
$$


In passing we note that if the degree of $P$ is sufficiently large then

$$
\sum_{k=1}^{n} A_{0 k n}(x) P\left(t_{k}\right)+\sum_{j=1}^{m-1} \sum_{k=1}^{n} P^{(j)}\left(t_{k}\right) A_{j k n}(x) \not \equiv P(x) .
$$

Define

$$
R(x):=s(x)+P(x) .
$$

Then, by the properties of the fundamental functions $A_{j k n}(x)$ in (2) we have, for $1 \leqslant k \leqslant n$

$$
R\left(t_{k}\right)=s\left(t_{k}\right)+P\left(t_{k}\right)=\phi\left(t_{k}\right)-P\left(t_{k}\right)+P\left(t_{k}\right)=\phi\left(t_{k}\right),
$$

and, for $1 \leqslant k \leqslant n, 1 \leqslant j \leqslant m-1$, we have

$$
R^{(j)}\left(t_{k}\right)=s^{(j)}\left(t_{k}\right)+P^{(j)}\left(t_{k}\right)=\phi^{(j)}\left(t_{k}\right)-P^{(j)}\left(t_{k}\right)+P^{(j)}\left(t_{k}\right)=0 .
$$

Also, we have

$$
\begin{aligned}
|R(x)-\phi(x)| & =|s(x)+P(x)-\phi(x)| \\
& \leqslant \sum_{k=1}^{n}\left|A_{0 k n}(x)\right| \cdot\|\phi-P\|+\sum_{j=1}^{m-1} \sum_{k=1}^{n}\left|A_{j k n}(x)\right| \cdot\left\|\phi^{(j)}-P^{(j)}\right\|+\|P-\phi\|
\end{aligned}
$$

and so

$$
\|R-\phi\| \leqslant\|\phi-P\|\left(1+\sum_{k=1}^{n}\left\|A_{0 k n}\right\|\right)+\sum_{j=1}^{m-1}\left\|\phi^{(j)}-P^{(j)}\right\|\left(\sum_{k=1}^{n}\left\|A_{j k n}\right\|\right) .
$$

Now remember that $m, n$ and the nodes $t_{1}, t_{2}, \ldots, t_{n}$ are all fixed. So we can appeal to Trigub's theorem on simultaneous approximation (see for example DeVore and Lorentz [3, Theorem 4.1, p. 245]) which guarantees that there is a polynomial $P$ such that

$$
\|\phi-P\|,\left\|\phi^{(1)}-P^{(1)}\right\|, \ldots,\left\|\phi^{(m-1)}-P^{(m-1)}\right\|
$$

can all be made arbitrarily small. Thus we can choose $P$ so that $\|R-\phi\| \leqslant \varepsilon$. This completes the proof of the Lemma.

REMARK. We are not saying anything about the degree of $R$.

LEMmA 4. If $n \geqslant 2$ and $1 \leqslant k \leqslant n-1$ then

$$
\frac{\sin \theta_{k, n}}{n\left|\cos \theta_{k, n}-\cos \theta_{k+1, n}\right|}>\frac{2}{3 \pi^{2}} \text {. }
$$


Proof: We shall write $\theta_{k}=\theta_{k, n}=(2 k-1) \pi /(2 n)$. Thus

$$
\begin{aligned}
E & :=\frac{\sin \theta_{k}}{n\left|\cos \theta_{k}-\cos \theta_{k+1}\right|} \\
& =\frac{\sin \theta_{k}}{2 n \sin \left(\left(\theta_{k}+\theta_{k+1}\right) / 2\right) \sin \left(\left(\theta_{k+1}-\theta_{k}\right) / 2\right)} \\
& >\frac{\sin \theta_{k}}{n\left(\theta_{k+1}-\theta_{k}\right) \sin \left(\left(\theta_{k}+\theta_{k+1}\right) / 2\right)} \\
& =\frac{1}{\pi} \frac{\sin \theta_{k}}{\sin \left(\left(\theta_{k}+\theta_{k+1}\right) / 2\right)} .
\end{aligned}
$$

We now consider three cases.

Case 1. Suppose that $\pi / 2<\theta_{k}<\theta_{k+1} \leqslant \pi$. Then $\sin \left(\left(\theta_{k}+\theta_{k+1}\right) / 2\right)<\sin \theta_{k}$. So

$$
E>\frac{1}{\pi} \geqslant \frac{2}{3 \pi^{2}} \text {. }
$$

Case 2. Suppose that $0<\theta_{k}<\theta_{k+1} \leqslant \pi / 2$. Then $\sin \left(\left(\theta_{k}+\theta_{k+1}\right) / 2\right)<\sin \theta_{k+1}$. So

$$
E \geqslant \frac{1}{\pi} \frac{\sin \theta_{k}}{\sin \theta_{k+1}} \geqslant \frac{1}{\pi} \frac{2 \theta_{k}}{\pi \theta_{k+1}}=\frac{2(2 k-1)}{\pi^{2}(2 k+1)}=\frac{2}{\pi^{2}}\left(1-\frac{2}{2 k+1}\right) \geqslant \frac{2}{3 \pi^{2}} .
$$

Case 3. Suppose that $\theta_{k}<\pi / 2<\theta_{k+1}$. Then $\left(\theta_{k}+\theta_{k+1}\right) / 2=\pi / 2$. So

$$
E \geqslant \frac{1}{\pi} \sin \theta_{k} \geqslant \frac{1}{\pi} \sin \pi / 4 \geqslant \frac{2}{3 \pi^{2}} .
$$

Thus, in any case, $E \geqslant 2 /\left(3 \pi^{2}\right)$.

LEMma 5. If $m \in\{1,3,5, \ldots\}$ and $p \in\{3,4,5, \ldots\}$, then there is a polynomial $R_{p}(x)\left(=R_{p, m}(x)\right)$ such that $\left\|R_{p}\right\| \leqslant 2$ and

$$
(\forall x \in[-\cos (\pi / p), \cos (\pi / p)])(\exists n>p)\left(\left|H_{m, n}\left(R_{p}, x\right)\right|>p\right) .
$$

ProOf: Let $q$ be a fixed natural number: during the course of proving this lemma, $q$ will be specified to be sufficiently large so that certain conditions are satisfied. To begin with, suppose that $q>p$.

We define the sets $S_{n}:=\left\{x_{1, n}, x_{2, n}, \ldots, x_{n, n}\right\}$, as in Lemma 1 , and

$$
S_{n}(a):=S_{n} \cap[-1, \cos a] \text {. }
$$

By part (i) of Lemma $1, S_{n} \cap S_{n+1}=\emptyset$ (that is, consecutive rows of the matrix of nodes $T$ in (1) have no common elements).

Now we shall construct a sequence of $q-1$ indices

$$
q=n_{1}<n_{2}<\ldots<n_{q-1}
$$


and begin the construction of a function $\phi:[-1,1] \rightarrow[-1,1]$.

Let $n_{1}=q$. Define $\phi$ on $S_{n_{1}} \cup S_{n_{1}+1}$ by

$$
\phi\left(x_{k, n_{1}}\right)= \begin{cases}0 & \text { if } x_{k, n_{1}} \in S_{n_{1}} \backslash S_{n_{1}}(\pi / q) \\ (-1)^{k-1} & \text { if } x_{k, n_{1}} \in S_{n_{1}}(\pi / q)\end{cases}
$$

and

$$
\phi\left(x_{k, n_{1}+1}\right)= \begin{cases}0 & \text { if } x_{k, n_{1}+1} \in S_{n_{1}+1} \backslash S_{n_{1}+1}(\pi / q) \\ (-1)^{k-1} & \text { if } x_{k, n_{1}+1} \in S_{n_{1}+1}(\pi / q) .\end{cases}
$$

We now find another pair of consecutive rows of the matrix $T$ which has almost nothing in common with the first pair of rows. To this end, let

$$
S=S_{n_{1}} \cup S_{n_{1}+1}
$$

and we apply part (ii) of Lemma 1 . Thus, there is $n_{2}>n_{1}$ such that

$$
S \cap\left(S_{n_{2}} \cup S_{n_{2}+1}\right)=\left(S \cap S_{n_{2}}\right) \cup\left(S \cap S_{n_{2}+1}\right)=\{0\} .
$$

We now define $\phi$ at all points of $S_{n_{2}} \cup S_{n_{2}+1}$ (with the possible exception of 0 in case it has been defined there already):

$$
\phi\left(x_{k, n_{2}}\right)= \begin{cases}0 & \text { if } x_{k, n_{2}} \in S_{n_{2}} \backslash S_{n_{2}}(2 \pi / q) \\ (-1)^{k-1} & \text { if } x_{k, n_{2}} \in S_{n_{2}}(2 \pi / q)\end{cases}
$$

and

$$
\phi\left(x_{k, n_{2}+1}\right)= \begin{cases}0 & \text { if } x_{k, n_{2}+1} \in S_{n_{2}+1} \backslash S_{n_{2}+1}(2 \pi / q) \\ (-1)^{k-1} & \text { if } x_{k, n_{2}+1} \in S_{n_{2}+1}(2 \pi / q) .\end{cases}
$$

We have now defined the two indices $q=n_{1}<n_{2}$, and defined $\phi$ on

$$
\left(S_{n_{1}} \cup S_{n_{1}+1}\right) \cup\left(S_{n_{2}} \cup S_{n_{2}+1}\right) .
$$

We continue this process and generate $n_{3}, n_{4}, \ldots, n_{q-1}$ as follows. Suppose that $i<q$ and that we have determined

$$
n_{1}, n_{2}, \ldots, n_{i-1} \text {. }
$$

By virtue of part (ii) of Lemma 1, we can choose $n_{\imath}>n_{i-1}$ so that the set $S_{n_{1}} \cup S_{n_{i}+1}$ has no point (or at most the point $\{0\}$ ) in common with the set

$$
\bigcup_{k=1}^{i-1}\left(S_{n_{k}} \cup S_{n_{k}+1}\right)
$$


We now define $\phi$ on the set $S_{n_{i}} \cup S_{n_{i}+1}$ (except at 0 if it has been defined there already):

$$
\phi\left(x_{k, n_{i}}\right)= \begin{cases}0 & \text { if } x_{k, n_{i}} \in S_{n_{i}} \backslash S_{n_{i}}(i \pi / q) \\ (-1)^{k-1} & \text { if } x_{k, n_{i}} \in S_{n_{i}}(i \pi / q)\end{cases}
$$

and

$$
\phi\left(x_{k, n_{i}+1}\right)= \begin{cases}0 & \text { if } x_{k, n_{i}+1} \in S_{n_{i}+1} \backslash S_{n_{i}+1}(i \pi / q) \\ (-1)^{k-1} & \text { if } x_{k, n_{i}+1} \in S_{n_{i}+1}(i \pi / q) .\end{cases}
$$

In this way, we generate $q-1$ indices $q=n_{1}<n_{2}<\ldots<n_{q-1}$ and define the function $\phi$ on all points of the set

$$
\tau_{p}=\bigcup_{i=1}^{q-1}\left(S_{n_{i}} \cup S_{n_{i}+1}\right)
$$

This set obviously depends on $q$ : but we shall show at the very end of the proof that $q$ is a function of $p$ and hence we denote the set by $\tau_{p}$.

Finally we extend the domain of $\phi$ and define $\phi$ throughout the interval $[-1,1]$ by requiring that

(i) $\phi(+1)=\phi(-1)=0$,

(ii) once all the nodes in $\tau_{p}$ have been ordered, the function $\phi$ should be monotonic between consecutive nodes - but not necessarily strictly monotonic, and

(iii) $\phi \in C^{m-1}[-1,1]$ and $\left(\forall t \in \tau_{p}\right)\left(1 \leqslant j \leqslant m-1 \Rightarrow \phi^{(j)}(t)=0\right)$.

Thus, we have $\phi \in C^{(m-1)}[-1,1]$ and $\|\phi\| \leqslant 1$. By Lemma 3 , there exists a polynomial $R$ such that

(i) $\left(\forall t \in \tau_{p}\right)(R(t)=\phi(t))$,

(ii) $\left(\forall t \in \tau_{p}\right)\left(1 \leqslant j \leqslant m-1 \Rightarrow R^{(j)}(t)=0\right)$, and

(iii) $\|R-\phi\| \leqslant 1$ and hence $\|R\| \leqslant 2$.

Hence $R$ satisfies the first requirement in the statement of the lemma: it remains to show that $R$ satisfies the second requirement in the lemma.

Assume that $x$ is fixed and $x \in[-\cos (\pi / p), \cos (\pi / p)]$. If $x=\cos \theta$ and $0 \leqslant \theta \leqslant \pi$ then $\theta \in[\pi / p, \pi-\pi / p]$. Then, because $q>p$, we can choose an index $i \in\{2,3, \ldots, q-1\}$ so that

$$
(i-1) \pi / q \leqslant \theta<i \pi / q
$$

By virtue of (5), corresponding to the index $i$, is an index $n_{i}$. Clearly

$$
p<q \leqslant n_{i} \leqslant n_{q-1}+1 .
$$


We shall now show that either $H_{m, n_{i}}(R, x)$ or $H_{m, n_{i}+1}(R, x)$ satisfies the second condition of the lemma. We consider these one at a time. $H_{m, n_{i}}(R, x)=\sum_{k=1}^{n_{i}} R\left(x_{k, n_{i}}\right) A_{0 k n_{i}}(x)=$ $\sum_{k=1}^{n_{i}} \phi\left(x_{k, n_{i}}\right) A_{0 k n_{i}}(x)$.

Define $r_{i}:=\min \left\{k: \theta_{k, n_{i}} \geqslant i \pi / q\right\}$, or equivalently,

$$
k \geqslant r_{i} \Leftrightarrow x_{k, n_{i}} \in S_{n_{i}}(i \pi / q) \Leftrightarrow i \pi / q \leqslant \theta_{k, n_{i}} \leqslant \pi
$$

Therefore

$$
H_{m, n_{i}}(R, x)=\sum_{k=r_{i}}^{n_{i}} \phi\left(x_{k, n_{i}}\right) A_{0 k n_{i}}(x) .
$$

There are two cases to consider arising from the possibility of $0 \in S_{n_{1}}$ and $\phi(0)$ having been defined earlier.

Case 1. If either $0 \notin S_{n_{i}}$ or $0 \in S_{n_{i}}$ and for all $1 \leqslant j<i, 0 \notin S_{n_{j}}$, then

$$
H_{m, n_{i}}(R, x)=\sum_{k=r_{i}}^{n_{i}}(-1)^{k-1} A_{0 k n_{i}}(x) .
$$

Case 2. If $0 \in S_{n_{i}}$ and for $1 \leqslant j<i$ we had $0 \in S_{n_{j}}$ then $\phi(0)$ would have been defined to be 0,1 or -1 before considering $S_{n_{i}}$. So, for some index $u$ we have

$$
H_{m, n_{i}}(R, x)=\sum_{k=r_{i}}^{n_{i}}(-1)^{k-1} A_{0 k n_{i}}(x)-(-1)^{u-1} A_{0 u n_{i}}(x)+\phi(0) A_{0 u n_{i}} .
$$

In either case, by Lemma 2, we have

$$
H_{m, n_{i}}(R, x)=\sum_{k=r_{i}}^{n_{i}}(-1)^{k-1} A_{0 k n_{i}}(x)+O(1)
$$

where $O(1)$ depends only on $m$.

We now use some ideas from Szabados [11, pp.359-360]. Remember that, here, $x_{k}=x_{k, n_{i}}$ and $\theta_{k}=\theta_{k, n_{i}}$.

$$
\sum_{k=r_{i}}^{n_{i}}(-1)^{k-1} A_{0 k n_{i}}(x)=\frac{\left(\cos n_{i} \theta\right)^{m}}{n_{i}^{m}} \sum_{k=r_{i}}^{n_{i}} \frac{\sin ^{m} \theta_{k}}{\left(\cos \theta-\cos \theta_{k}\right)^{m}} B_{0 k n_{i}}(x)
$$

where

$$
B_{0 k n_{i}}(x) \geqslant c_{1}\left(\frac{x-x_{k}}{x_{k}-x_{k \pm 1}}\right)^{m-1}>0
$$


with only one of the signs in $k \pm 1$ applying in any term. Note that by (6), (8) $\theta<i \pi / q \leqslant$ $\theta_{k}$. So we have:

$$
\begin{aligned}
& \left|\sum_{k=r_{i}}^{n_{i}}(-1)^{k-1} A_{0 k n_{i}}(x)\right| \\
& \quad \geqslant c_{1} \frac{\left|\cos n_{i} \theta\right|^{m}}{n_{i}} \sum_{k=r_{i}}^{n_{i}} \frac{\sin \theta_{k}}{\left(\cos \theta-\cos \theta_{k}\right)}\left(\frac{\sin \theta_{k}}{n_{i}\left(\cos \theta_{k}-\cos \theta_{k \pm 1}\right)}\right)^{m-1} \\
& \quad \geqslant c_{2} \frac{\left|\cos n_{i} \theta\right|^{m}}{n_{i}} \sum_{k=r_{i}}^{n_{i}} \frac{\sin \theta_{k}}{\left(\cos \theta-\cos \theta_{k}\right)}
\end{aligned}
$$

the last step following from Lemma 4 and $0<\left(\cos \theta-\cos \theta_{k}\right)^{m-1}<2^{m-1}$.

At this point we can follow Natanson [7, pp. 41-43] exactly. For completeness we reproduce his calculations.

For fixed $\theta$, the function $(\sin t) /(\cos \theta-\cos t)$ is decreasing for $t \in(\theta, \pi)$ since its derivative is

$$
\frac{\cos \theta \cos t-1}{(\cos \theta-\cos t)^{2}}<0
$$

Remember that we write $\theta_{k}$ for $\theta_{k, n_{i}}$. For $\theta<\theta_{k}<t<\theta_{k+1}<\pi$ we have

$$
\frac{\sin \theta_{k}}{\cos \theta-\cos \theta_{k}}>\frac{\sin t}{\cos \theta-\cos t} \text {. }
$$

For $k=r_{i}, r_{i}+1, \ldots, n_{i}-1$, we have $\theta<\theta_{k}<\theta_{k+1}<\pi$, and hence

$$
\frac{\pi}{n_{i}} \frac{\sin \theta_{k}}{\cos \theta-\cos \theta_{k}}>\int_{\theta_{k}}^{\theta_{k+1}} \frac{\sin t}{\cos \theta-\cos t} d t
$$

Also,

$$
\frac{\pi}{2 n_{i}} \frac{\sin \theta_{n_{i}}}{\cos \theta-\cos \theta_{n_{i}}}>\int_{\theta_{n_{1}}}^{\pi} \frac{\sin t}{\cos \theta-\cos t} d t
$$

Therefore,

$$
\begin{aligned}
& \frac{\pi}{n_{i}} \sum_{k=r_{i}}^{n_{i}} \frac{\sin \theta_{k}}{\cos \theta-\cos \theta_{k}}>\int_{\theta_{r_{i}}}^{\pi} \frac{\sin t}{\cos \theta-\cos t} d t \\
& \frac{1}{n_{i}} \sum_{k=r_{i}}^{n_{i}} \frac{\sin \theta_{k}}{\cos \theta-\cos \theta_{k}}>\frac{1}{\pi} \ln \frac{1+\cos \theta}{\cos \theta-\cos \theta_{r_{i}}}>0 .
\end{aligned}
$$

Now recall that $\pi / p \leqslant \theta \leqslant \pi-\pi / p$. So

$$
1+\cos \theta \geqslant 1-\cos \pi / p \text {. }
$$

On the other hand,

$$
0<\cos \theta-\cos \theta_{r_{i}} \leqslant \theta_{r_{i}}-\theta=\left(\frac{\left(2 r_{i}-1\right) \pi}{2 n_{i}}-\frac{i \pi}{q}\right)+\left(\frac{i \pi}{q}-\theta\right) .
$$


Recall that

$$
r_{i}=\min \left\{k: 1 \leqslant k \leqslant n_{i}, \frac{i \pi}{q} \leqslant \frac{(2 k-1) \pi}{2 n_{i}}\right\} .
$$

So

$$
0 \leqslant \frac{\left(2 r_{i}-1\right) \pi}{2 n_{i}}-\frac{i \pi}{q} \leqslant \frac{\pi}{n_{i}} \leqslant \frac{\pi}{q} .
$$

Also, by (6),

$$
i \pi / q-\theta \leqslant \pi / q
$$

Therefore, by (13), (14), (15),

$$
0<\cos \theta-\cos \theta_{\tau_{i}} \leqslant \frac{2 \pi}{q} .
$$

So, by (10), (11), (12) and (16),

$$
\left|\sum_{k=r_{i}}^{n_{i}}(-1)^{k-1} A_{0 k n_{i}}\right| \geqslant c_{3}\left|\cos n_{i} \theta\right|^{m} \ln \left(\frac{(1-\cos (\pi / p)) q}{2 \pi}\right) .
$$

Similarly we could have obtained

$$
\left|\sum_{k=r_{i}}^{n_{2}+1}(-1)^{k-1} A_{0 k\left(n_{i}+1\right)}\right| \geqslant c_{3}\left|\cos \left(n_{i}+1\right) \theta\right|^{m} \ln \left(\frac{(1-\cos (\pi / p)) q}{2 \pi}\right) .
$$

Since $\pi / p \leqslant \theta \leqslant \pi-\pi / p$ and $p>2$ it follows that $\sin \theta \geqslant \sin (\pi / p) \geqslant 2 / p$. This, together with $\sin \theta=\sin (n+1) \theta \cos n \theta-\sin n \theta \cos (n+1) \theta$, leads to

$$
2 / p \leqslant \sin \theta \leqslant\left|\cos n_{i} \theta\right|+\left|\cos \left(n_{i}+1\right) \theta\right| .
$$

Thus, either $\left|\cos n_{i} \theta\right| \geqslant 1 / p$ (in which case define $n:=n_{i}$ ) or $\left|\cos \left(n_{i}+1\right) \theta\right| \geqslant 1 / p$ (in which case define $\left.n:=n_{i}+1\right)$. So, with the appropriate definition of $n$ we have

$$
\left|\sum_{k=r_{i}}^{n}(-1)^{k-1} A_{0 k n}\right| \geqslant \frac{c_{5}}{p^{m}} \ln \left(\frac{(1-\cos (\pi / p)) q}{2 \pi}\right) .
$$

Now $m$ and $p$ may be regarded as fixed. By (17) we can choose $q$ large enough so that

$$
\left|\sum_{k=r_{i}}^{n}(-1)^{k-1} A_{0 k n}\right|
$$

could be made arbitrarily large. Hence, by recalling (9) we can choose $q(>p)$ to be the smallest natural number such that $\left|H_{m, n}(R, x)\right|>p$ and this completes the proof of the Lemma.

REMARK. The last sentence in the above proof ensures that $q$ is determined uniquely by $p$. 


\section{Proof of TheOREM 2 in $(-1,1)$}

In this section we prove Theorem 2 only for $x$ in the open interval $(-1,1)$.

Assume that the matrix of nodes is given by $T$ as in (1) and that $m \in\{1,3,5, \ldots\}$. By (3), we define the Lebesgue constant associated with the operator $H_{m, n}$ by

$$
\lambda_{n}:=\max \left\{\sum_{k=1}^{n}\left|A_{0 k n}(x)\right|:-1 \leqslant x \leqslant 1\right\} .
$$

So, if $g \in C[-1,1], x \in[-1,1]$ and $n \in \mathbf{Z}^{+}$, then

$$
\left|H_{m, n}(g, x)\right| \leqslant \lambda_{n}\|g\| .
$$

We shall define a function $f$ which will prove to be the function mentioned in the statement of Theorem 2 (at least for $(-1,1)$ ). For each $p \in\{3,4,5, \ldots\}$, construct a polynomial $R_{p}$ in accordance with Lemma 5 and let $r(p):=$ degree $R_{p}$. For each $x \in$ $[-\cos (\pi / p), \cos (\pi / p)]$, let $n(x, p)>p$ be an index such that $\left|H_{m, n(x, p)}\left(R_{p}, x\right)\right|>p$. The existence of $n(x, p)$ is assured by Lemma 5 . By (7)

$$
p<n(x, p) \leqslant n_{q-1}+1=: N(p)
$$

(since $q$ is a function of $p$ ). Choose an infinite sequence $\left\{p_{1}, p_{2}, \ldots\right\}$ of natural numbers such that

$$
\left.\begin{array}{l}
p_{1}=3 \\
p_{k+1}>\max \left\{m r\left(p_{i}\right)^{2 m-1}: 1 \leqslant i \leqslant k\right\} \\
p_{k+1}>p_{k}^{2} \\
p_{k+1}>\max \left\{\lambda_{p_{k}}^{2}, \lambda_{p_{k}+1}^{2}, \ldots, \lambda_{N\left(p_{k}\right)}^{2}\right\} .
\end{array}\right\}
$$

Finally, define $f:[-1,1] \rightarrow \mathbf{R}$ by

$$
f(x):=\sum_{k=1}^{\infty} \frac{R_{p_{k}}(x)}{\sqrt{ } p_{k}}
$$

It remains to show that $f$ has the required properties.

It will be useful to define the quantity

$$
S:=\sum_{k=1}^{\infty} \frac{2}{\sqrt{ } p_{k}}
$$

From the third condition in (21), it follows that $S$ is finite. Now observe that the series in (22) is absolutely convergent because, by Lemma 5 and (23)

$$
\sum_{k=1}^{\infty} \frac{\left|R_{p_{k}}(x)\right|}{\sqrt{ } p_{k}} \leqslant \sum_{k=1}^{\infty} \frac{2}{\sqrt{ } p_{k}}=S
$$


Thus $f$, as given by (22), is a well defined element of $C[-1,1]$.

Now let $x_{0} \in(-1,1)$ and $K>0$. We must find a number $n$ such that $\left|H_{m, n}\left(f, x_{0}\right)\right|$ $>K$.

Since $x_{0} \in(-1,1)$, we can choose $t$ to be so large that

$$
-\cos \frac{\pi}{p_{t}} \leqslant x_{0} \leqslant \cos \frac{\pi}{p_{t}}
$$

Let $n=n\left(x_{0}, p_{t}\right)$. Then, by (22), we can write

$$
\begin{aligned}
f(x) & =\sum_{k=1}^{t-1} \frac{R_{p_{k}}(x)}{\sqrt{ } p_{k}}+\frac{R_{p_{t}}(x)}{\sqrt{ } p_{t}}+\sum_{k=t+1}^{\infty} \frac{R_{p_{k}}(x)}{\sqrt{ } p_{k}} \\
& =A(x)+\frac{R_{p_{t}}(x)}{\sqrt{ } p_{t}}+B(x), \text { say. }
\end{aligned}
$$

To see the effect of the linear operator $H_{m, n}$ on $f$, we shall examine its effect on each of the three terms on the right hand side of (25) one at a time.

If we consider the third term in (25), then by (19), Lemma $5,(21)$ and (23) we obtain

$$
\begin{aligned}
\left|H_{m, n}(B, x)\right| & \leqslant \lambda_{n}\|B\| \\
& \leqslant \lambda_{n} \sum_{k=t+1}^{\infty} \frac{2}{\sqrt{ } p_{k}}=\lambda_{n} \sum_{k=1}^{\infty} \frac{2}{\sqrt{ } p_{t+k}} \\
& <\lambda_{n}\left(\frac{2}{\sqrt{ } p_{t+1}}+\sum_{k=2}^{\infty} \frac{2}{\sqrt{ }\left(p_{t+(k-1)}^{2}\right)}\right) \\
& <\lambda_{n}\left(\frac{2}{\sqrt{ } p_{t+1}}+\sum_{k=2}^{\infty} \frac{2}{\sqrt{ }\left(p_{t+(k-1)} p_{t+1}\right)}\right) \\
& \leqslant \frac{\lambda_{n}(2+S)}{\sqrt{ } p_{t+1}} .
\end{aligned}
$$

So, by (20) and (21),

$$
\left|H_{m, n}\left(B, x_{0}\right)\right|<2+S
$$

If we consider the first term in (25), we obtain

$$
H_{m, n}(A, x)=\sum_{k=1}^{t-1} H_{m, n}\left(R_{p_{k}}, x\right) / \sqrt{ } p_{k} .
$$

By (3)

$$
H_{m, n}\left(R_{p_{k}}, x\right)=\sum_{i=1}^{n} R_{p_{k}}\left(x_{i, n}\right) A_{0 i n}(x)
$$

Now (7) and (21) imply that

$$
n=n\left(x_{0}, p_{t}\right)>p_{t}>\max \left\{r\left(p_{1}\right), r\left(p_{2}\right), \ldots, r\left(p_{t-1}\right)\right\}
$$


and hence $m n-1>\max \left\{r\left(p_{1}\right), r\left(p_{2}\right), \ldots, r\left(p_{t-1}\right)\right\}$. So, for $1 \leqslant k \leqslant t-1$

$$
R_{p_{k}}(x) \equiv H_{m, n}\left(R_{p_{k}}, x\right)+\sum_{j=1}^{m-1} \sum_{i=1}^{n} R^{(j)}\left(x_{i, n}\right) A_{j i n}(x)
$$

and therefore

$$
H_{m, n}\left(R_{p_{k}}, x\right) \equiv R_{p_{k}}(x)-\sum_{j=1}^{m-1} \sum_{i=1}^{n} R^{(j)}\left(x_{i, n}\right) A_{j i n}(x) .
$$

Using Markov's inequality, Szabados [11, Theorem 2], (21) and $\left\|R_{p_{k}}\right\| \leqslant 2$ we obtain, for $1 \leqslant k \leqslant t-1$,

$$
\begin{aligned}
\left|H_{m, n}\left(R_{p_{k}}, x\right)\right| & \leqslant\left\|R_{p_{k}}\right\|+\sum_{j=1}^{m-1} \sum_{i=1}^{n}\left\|R_{p_{k}}\right\| r\left(p_{k}\right)^{2 j}\left|A_{j i n}(x)\right| \\
& \leqslant\left\|R_{p_{k}}\right\|\left(1+\sum_{j=1}^{m-1} \sum_{i=1}^{n} r\left(p_{k}\right)^{2 j} \frac{\left|A_{j i n}(x)\right|}{\left(1-x_{i}^{2}\right)^{j / 2}}\right) \\
& \leqslant\left\|R_{p_{k}}\right\|\left(1+\sum_{j=1}^{m-1} r\left(p_{k}\right)^{2 j}\left(\left\|\sum_{i=1}^{n} \frac{\left|A_{j i n}(x)\right|}{\left(1-x_{i}^{2}\right)^{j / 2}}\right\|\right)\right) \\
& \leqslant\left\|R_{p_{k}}\right\|\left(1+\frac{m-1}{n} r\left(p_{k}\right)^{2 m-2}\right) \\
& \leqslant\left\|R_{p_{k}}\right\|\left(1+\frac{m r\left(p_{k}\right)^{2 m-1}}{n r\left(p_{k}\right)}\right) \\
& \leqslant\left\|R_{p_{k}}\right\|\left(1+\frac{p_{k+1}}{n r\left(p_{k}\right)}\right) \\
& \leqslant\left\|R_{p_{k}}\right\|\left(1+\frac{p_{t}}{n r\left(p_{k}\right)}\right) \leqslant 4 .
\end{aligned}
$$

This inequality, (27) and (23) imply that

$$
\left|H_{m, n}\left(A, x_{0}\right)\right| \leqslant \sum_{k=1}^{t-1} 4 / \sqrt{ } p_{k} \leqslant 2 S .
$$

Finally we consider the second term in (25) which, as we shall see, is the dominant term. By the definition of $n:=n\left(x_{0}, p_{t}\right)$,

$$
\left|H_{m, n}\left(\frac{R_{p_{t}}}{\sqrt{ } p_{t}}, x_{0}\right)\right|=\frac{\left|H_{m, n}\left(R_{p_{t}}, x_{0}\right)\right|}{\sqrt{ } p_{t}}>\sqrt{ } p_{t}
$$

and, according to (24), we can make $p_{t}$ as large as we please if $x_{0}$ is in the open interval $(-1,1)$.

From (25), (26), (28) and (29), it follows that for any $K>0$ we can find $n$ such that $\left|H_{m, n}\left(f, x_{0}\right)\right|>K$. Therefore, we have shown that, for all $x \in(-1,1)$,

$$
\limsup _{n \rightarrow \infty}\left|H_{m, n}(f, x)\right|=+\infty
$$




\section{Proof of Theorem 2 in $[-1,1]$}

In this section we shall complete the proof of Theorem 2. We employ the general approach suggested by Grünwald [5] and Marcinkiewicz [6] and supplement it with technical results from Szabados [11].

As before, we assume that $m \in\{1,3,5, \ldots\}$ is fixed.

In the previous section, we have shown that the function $f \in C([-1,1])$ as defined in (22) satisfies (30). There are four cases to be considered.

CASE 1: If (30) holds for all $x \in[-1,1]$ then we have proved Theorem 2 completely.

CASE 2: If (30) holds for all $x \in[-1,1)$ but not for $x=1$, then we proceed as follows. Construct a function $\psi$ such that, for all $x \in[-1,1)$ the sequence $\left\{H_{m, n}(\psi, x): n=\right.$ $1,2,3, \ldots\}$ is bounded, but the sequence $\left\{H_{m, n}(\psi,+1): n=1,2,3, \ldots\right\}$ is unbounded. Then the sequence $\left\{H_{m, n}(f+\psi, x): n=1,2,3, \ldots\right\}$ diverges for all $x \in[-1,1]$ and we have proved Theorem 2 completely.

CASE 3: If (30) holds for all $x \in(-1,1]$ but not for $x=-1$ then we proceed in a manner similar to that used in Case 2 .

CASE 4: If $(30)$ holds for all $x \in(-1,1)$ but not for $x= \pm 1$ then we combine the approaches in Case 2 and Case 3.

So it suffices to consider Case 2. To this end we shall construct a function $\psi \in$ $C([-1,1])$ such that

$$
(\forall x \in[-1,1))\left(\left\{H_{m, n}(\psi, x): n=1,2,3, \ldots\right\} \text { is bounded }\right)
$$

and

$$
\limsup _{n \rightarrow \infty}\left|H_{m, n}(\psi, 1)\right|=+\infty
$$

The following subsections are devoted to defining $\psi$ and proving that $\psi$ satisfies (31) and (32).

STEP 1. Firstly, we construct the function $\psi$. We shall need an infinite sequence of natural numbers $n_{1}<n_{2}<n_{3}<\ldots$ which increase sufficiently rapidly for our purposes.

We shall require that if

$$
k_{i}:=\left[\frac{n_{i}^{3 / 4}}{\log n_{i}} \cdot \frac{2}{\pi}\right] \quad(i=1,2,3, \ldots)
$$

and

$$
K_{i}:=\left[n_{i}^{3 / 4} \cdot \frac{2}{\pi}\right] \quad(i=1,2,3, \ldots)
$$


then

$$
x_{k_{i}, n_{i}}<x_{K_{i+1}, n_{i+1}} \quad(i=1,2,3, \ldots) .
$$

(To construct such a sequence, we could choose $n_{1}=10, k_{1}=1, K_{1}=3$. Once we have chosen $n_{1}, n_{2}, \ldots, n_{i}$ we can choose $n_{i+1}$ to ensure that (33) is satisfied.)

Note that if

$$
I_{i}=\left[x_{K_{i}, n_{i}}, x_{k_{i}, n_{i}}\right] \quad(i=1,2,3, \ldots)
$$

then $I_{i} \cap I_{j}=\emptyset$ whenever $i \neq j$.

Later we shall demand that the sequence $\left\{n_{1}, n_{2}, n_{3}, \ldots\right\}$ satisfies other conditions as well - but these will serve only to force the sequence to diverge more rapidly.

For each index $i \geqslant 1$, define the function $\psi_{i}:[-1,1] \rightarrow[-1,1]$ which satisfies the following four conditions. First,

$$
\psi_{i}\left(x_{k, n_{i}}\right):= \begin{cases}0 & \text { if } k \leqslant k_{i} \\ (-1)^{k-1} & \text { if } k_{i}+1 \leqslant k \leqslant K_{i}-1 \\ 0 & \text { if } k \geqslant K_{i} .\end{cases}
$$

Second,

$$
x \notin I_{i} \Rightarrow \psi_{i}(x):=0
$$

Third, $\left\|\psi_{i}\right\| \leqslant 1$. Fourth, for some constant $M_{i}>0, \psi_{i}^{(m-1)} \in \operatorname{Lip}_{M_{i}} 1$.

Recall the definition of the Lebesgue constants $\lambda_{n}(n=1,2,3, \ldots)$ in (18). Define

$$
\rho_{i}:= \begin{cases}1 & \text { if } i=0 \\ \max \left\{\lambda_{n_{1}}, \lambda_{n_{2}}, \ldots, \lambda_{n_{i}}\right\} & \text { if } i \geqslant 1 .\end{cases}
$$

Now define the function $\psi:[-1,1] \rightarrow \mathbf{R}$ by

$$
\psi(x):=\sum_{i=1}^{\infty} \frac{\psi_{i}(x)}{i^{2} \rho_{i-1}} .
$$

We know from Szabados [11, Theorems 1, 2] that $\lambda_{n} \sim \log n($ as $n \rightarrow \infty)$. So we can choose our sequence $n_{1}<n_{2}<n_{3}<\ldots$ to ensure that

$$
\sum_{i=1}^{\infty} \frac{1}{i^{2} \rho_{i-1}}<2
$$

Hence

$$
\|\psi\| \leqslant \sum_{i=1}^{\infty} \frac{\left\|\psi_{i}\right\|}{i^{2} \rho_{i-1}}<2
$$


STEP 2. Having defined the function $\psi$, our next step is to prove that $\psi$ satisfies (31).

Let $x_{0} \in[-1,1)$.

Define $J_{0}:=I_{0}=\left[-1, x_{K_{1}, n_{1}}\right)$ and, for $i \geqslant 1, J_{i}:=\left[x_{K_{i}, n_{i}}, x_{K_{i+1}, n_{i+1}}\right)$. From the construction of the intervals $I_{i}$ and this definition, it follows that $0 \leqslant i<j \Rightarrow J_{i} \cap J_{j}=\emptyset$ and that $[-1,1)=\bigcup_{i=0}^{\infty} J_{i}$. Thus the intervals $\left\{J_{0}, J_{1}, J_{2}, \ldots\right\}$ partition the interval $[-1,1)$.

Therefore, $(\exists ! s \geqslant 0)\left(x_{0} \in J_{s}\right)$. Hence we write

$$
\psi(x)=\sum_{i=1}^{\infty} \frac{\psi_{i}(x)}{i^{2} \rho_{i-1}}=\sum_{i=1}^{s} \frac{\psi_{i}(x)}{i^{2} \rho_{i-1}}+\sum_{i=s+1}^{\infty} \frac{\psi_{i}(x)}{i^{2} \rho_{i-1}}=\phi_{s}(x)+\tau_{s}(x), \quad \text { say }
$$

(If $s=0$ then $\phi_{s} \equiv 0$.)

Since $\psi_{i}^{(m-1)} \in \operatorname{Lip}_{M_{i}} 1(1 \leqslant i \leqslant s)$ we have $\phi_{s}^{(m-1)} \in \operatorname{Lip}_{M_{s}{ }^{1}}$ for some positive constant $M_{s}^{\prime}$. Therefore by [3, Theorem 4.8, p. 251] there exists $c_{s} \geqslant 0$ such that for all $n \in \mathrm{N}$ there is a polynomial $R_{n}=R_{n}\left(\phi_{s}\right)$ of degree at most $n$ such that for all $x=\cos \theta \in[-1,1\}$ and for all $t \in\{0,1,2, \ldots, m-1\}$ we have

$$
\left|\phi_{s}^{(t)}(x)-R_{n}^{(t)}(x)\right| \leqslant c_{s}\left(\frac{\sin \theta}{n}\right)^{m-t}
$$

Also

$$
\left\|\phi_{s}\right\| \leqslant\|\psi\|<2
$$

We now estimate $\left|H_{m, n}\left(\phi_{s}, x_{0}\right)-\phi_{s}\left(x_{0}\right)\right|$ using (38) and the fact that the higher order Hermite interpolation operator reproduces polynomials of the appropriate degree.

$$
\begin{aligned}
& H_{m, n}\left(\phi_{s}, x_{0}\right)-\phi_{s}\left(x_{0}\right) \\
& \quad=H_{m, n}\left(\phi_{s}, x_{0}\right)-R_{n-1}\left(x_{0}\right)+R_{n-1}\left(x_{0}\right)-\phi_{s}\left(x_{0}\right) \\
& =H_{m, n}\left(\phi_{s}, x_{0}\right)-\left(H_{m, n}\left(R_{n-1}, x_{0}\right)+\sum_{t=1}^{m-1} \sum_{k=1}^{n} R_{n-1}^{(t)}\left(x_{k, n}\right) A_{t k n}\left(x_{0}\right)\right)+R_{n-1}\left(x_{0}\right)-\phi_{s}\left(x_{0}\right) \\
& =H_{m, n}\left(\phi_{s}-R_{n-1}, x_{0}\right)-\sum_{t=1}^{m-1} \sum_{k=1}^{n} R_{n-1}^{(t)}\left(x_{k, n}\right) A_{t k n}\left(x_{0}\right)+R_{n-1}\left(x_{0}\right)-\phi_{s}\left(x_{0}\right) .
\end{aligned}
$$

Therefore

$$
\begin{aligned}
& \left|H_{m, n}\left(\phi_{s}, x_{0}\right)-\phi_{s}\left(x_{0}\right)\right| \\
& \quad \leqslant\left|H_{m, n}\left(\phi_{s}-R_{n-1}, x_{0}\right)\right|+\sum_{t=1}^{m-1} \sum_{k=1}^{n}\left|R_{n-1}^{(t)}\left(x_{k, n}\right) A_{t k n}\left(x_{0}\right)\right|+\left|R_{n-1}\left(x_{0}\right)-\phi_{s}\left(x_{0}\right)\right| \\
& \quad=s_{1}\left(x_{0}\right)+s_{2}\left(x_{0}\right)+s_{3}\left(x_{0}\right) \quad \text { say. }
\end{aligned}
$$

We now estimate each of $s_{1}\left(x_{0}\right), s_{2}\left(x_{0}\right), s_{3}\left(x_{0}\right)$ in turn. 
To estimate $s_{1}\left(x_{0}\right)$ we use (38) and $[11$, p. 367, (27), (28)]. In the calculation which follows, the $O(1)$ term may depend on $s$ and hence on $x_{0}$. Also, the index $j_{0}$ is defined by

$$
\begin{aligned}
\left|x_{0}-x_{j_{0}, n}\right|=\min \left\{\left|x_{0}-x_{k, n}\right|: k=1,2, \ldots, n\right\} \\
s_{1}\left(x_{0}\right)=\left|\sum_{k=1}^{n}\left(\phi_{s}\left(x_{k, n}\right)-R_{n-1}\left(x_{k, n}\right)\right) A_{0 k n}\left(x_{0}\right)\right| \\
\leqslant \sum_{k=1}^{n}\left|\phi_{s}\left(x_{k, n}\right)-R_{n-1}\left(x_{k, n}\right)\right| \cdot\left|A_{0 k n}\left(x_{0}\right)\right| \\
=O(1) \sum_{k=1}^{n}\left(\frac{\sin \theta_{k, n}}{n-1}\right)^{m} \cdot\left|A_{0 k n}\left(x_{0}\right)\right| \\
=O(1) \sum_{k=1}^{n}\left(\frac{\sin \theta_{k, n}}{n-1}\right)^{m} \cdot \frac{1}{\left|j_{0}-k\right|+1} \\
=O(1) n^{-m} \log n \quad \text { as } n \rightarrow \infty .
\end{aligned}
$$

To estimate $s_{2}\left(x_{0}\right)$ we again call on $[11$, p. $367,(27),(28)]$ and use the fact that if $1 \leqslant t \leqslant m-1$ then

$$
\left|R_{n-1}^{(t)}(x)\right| \leqslant\left|R_{n-1}^{(t)}(x)-\phi_{s}^{(t)}(x)\right|+\left|\phi_{s}^{(t)}(x)\right|=O(1)\left(\left(\frac{\sin \theta}{n-1}\right)^{m-t}+1\right)=O(1)
$$

where $O(1)$ may depend on $s$ and hence $x_{0}$. Therefore

$$
\begin{aligned}
s_{2}\left(x_{0}\right) & =\sum_{\substack{t=1 \\
t \text { odd }}}^{m-1} \sum_{k=1}^{n}\left|R_{n-1}^{(t)}\left(x_{k, n}\right)\right| \cdot\left|A_{t k n}\left(x_{0}\right)\right|+\sum_{\substack{t=1 \\
t=1 \\
\text { even }}}^{m-1} \sum_{k=1}^{n}\left|R_{n-1}^{(t)}\left(x_{k, n}\right)\right| \cdot\left|A_{t k n}\left(x_{0}\right)\right| \\
& =O(1) \sum_{\substack{t=1 \\
t \text { odd } \\
\text { od }}}^{m-1} \sum_{k=1}^{n}\left|A_{t k n}\left(x_{0}\right)\right|+O(1) \sum_{\substack{t=1 \\
t \text { odd }}}^{n-1} \sum_{k=1}^{n}\left|A_{t k n}\left(x_{0}\right)\right| \\
& =O(1) \sum_{\substack{t=1 \\
t \text { odd }}}^{n-1} \sum_{k=1}^{n}\left(\frac{\sin \theta_{k, n}}{n}\right)^{t} \cdot \frac{1}{1+\left|k-j_{0}\right|^{2}}+O(1) \sum_{\substack{t=1 \\
t \text { even }}}^{m-1} \sum_{k=1}^{n}\left(\frac{\sin \theta_{k, n}}{n}\right)^{t} \cdot \frac{1}{1+\left|k-j_{0}\right|} \\
& =O(1) n^{-1}+O(1) n^{-2} \log n \\
& =O(1) n^{-1} \quad \text { as } n \rightarrow \infty .
\end{aligned}
$$

To estimate $s_{3}\left(x_{0}\right)$ we use (38) to obtain

$$
s_{3}\left(x_{0}\right)=\left|R_{n-1}\left(x_{0}\right)-\phi_{s}\left(x_{0}\right)\right|=O(1) n^{-m} \quad \text { as } n \rightarrow \infty
$$


Remembering that $x_{0}, s$ are fixed and (39), and using (41), (42), (43) in (40), we obtain

$$
\begin{aligned}
\left|H_{m, n}\left(\phi_{s}, x_{0}\right)\right| & \leqslant\left|\phi_{s}\left(x_{0}\right)\right|+\left|H_{m, n}\left(\phi_{s}, x_{0}\right)-\phi_{s}\left(x_{0}\right)\right| \\
& \leqslant 2+O(1)\left(n^{-m} \log n+n^{-1}+n^{-m}\right) \\
& \leqslant 3 \text { if } n \text { is sufficiently large. }
\end{aligned}
$$

Next we estimate $H_{m, n}\left(\tau_{s}, x_{0}\right)$ where $\tau_{s}$ is defined in (37). Obviously $H_{m, n}\left(\tau_{s}, x_{0}\right)=$ $\sum_{k=1}^{n} \tau_{s}\left(x_{k, n}\right) A_{0 k n}\left(x_{0}\right)$; hence, to calculate $H_{m, n}\left(\tau_{s}, x_{0}\right)$ requires $\tau_{s}\left(x_{k, n}\right)$ which, in turn, requires $\psi_{i}\left(x_{k, n}\right)(i \geqslant s+1)$ by $(37)$.

Notice that, by (34), $\left(\psi_{i}\left(x_{k, n}\right) \neq 0\right) \Rightarrow\left(x \in I_{i}\right)$. Therefore, if for some $i \geqslant s+1$ we have $\psi_{i}\left(x_{k, n}\right) \neq 0$ then $x_{k, n} \in I_{i}$; that is $x_{K_{i}, n_{i}} \leqslant x_{k, n} \leqslant x_{k_{i}, n_{i}}$ and hence $x_{k, n}-x_{0} \geqslant$ $x_{K_{s+1}, n_{s+1}}-x_{0}:=\delta\left(x_{0}\right)>0$. Define $y_{s}:=x_{K_{s+1}, n_{s+1}}$. Then we have shown that

$$
\left(x_{k, n}<y_{s}\right) \Rightarrow\left((\forall i \geqslant s+1)\left(\psi_{i}\left(x_{k, n}\right)=0\right) \Rightarrow\left(\tau_{s}\left(x_{k, n}\right)=0\right)\right. \text {. }
$$

This allows us to write $\left|H_{m, n}\left(\tau_{s}, x_{0}\right)\right| \leqslant 2 \sum_{x_{k, n} \geqslant y_{s}}\left|A_{0 k n}\left(x_{0}\right)\right|$. We use Szabados $[\mathbf{1 1}$, p. 367, $(27)]$.

$$
\begin{aligned}
\left|H_{m, n}\left(\tau_{s}, x_{0}\right)\right| & \leqslant 2 \sum_{x_{k, n} \geqslant y_{s}}\left|A_{0 k n}\left(x_{0}\right)\right| \\
& =O(1) \sum_{x_{k, n} \geqslant y_{s}}\left(\frac{\sin \theta_{k}}{n\left(x_{k, n}-x_{0}\right)}\right)^{m} \sum_{i=0}^{m}\left(\frac{n}{\sin \theta_{k}}\right)^{i} \cdot\left(x_{k}-x_{0}\right)^{i} \\
& =O(1) \sum_{x_{k, n} \geqslant y_{s}} \frac{1}{n\left(x_{k, n}-x_{0}\right)^{m}} \\
& =O(1) \delta\left(x_{0}\right)^{-m} \\
& =O(1) \text { where, again, } O(1) \text { depends on } x_{0} .
\end{aligned}
$$

Recalling (37), (44) and (45), we get, as $n \rightarrow \infty$,

$$
\left|H_{m, n}\left(\psi, x_{0}\right)\right|=\left|H_{m, n}\left(\phi_{s}, x_{0}\right)+H_{m, n}\left(\tau_{s}, x_{0}\right)\right| \leqslant\left|H_{m, n}\left(\phi_{s}, x_{0}\right)\right|+\left|H_{m, n}\left(\tau_{s}, x_{0}\right)\right|=O(1) .
$$

Hence $\psi$ satisfies (31).

STEP 3. The final step is to prove that $\psi$ satisfies (32). We prove that the sequence $\left\{H_{m, n_{j}}(\psi,+1): j \in \mathrm{Z}^{+}\right\}$is unbounded.

Let $j \geqslant 1$. Then

$$
\psi(x)=\sum_{i=1}^{j-1} \frac{\psi_{i}(x)}{i^{2} \rho_{i-1}}+\frac{\psi_{j}(x)}{j^{2} \rho_{j-1}}+\sum_{i=j+1}^{\infty} \frac{\psi_{i}(x)}{i^{2} \rho_{i-1}}
$$


and hence

$$
\begin{aligned}
& H_{m, n_{j}}(\psi, 1) \\
& \quad=H_{m, n_{j}}\left(\sum_{i=1}^{j-1} \frac{\psi_{i}(\cdot)}{i^{2} \rho_{i-1}}, 1\right)+H_{m, n_{j}}\left(\frac{\psi_{j}(\cdot)}{j^{2} \rho_{j-1}}, 1\right)+H_{m, n_{j}}\left(\sum_{i=j+1}^{\infty} \frac{\psi_{i}(\cdot)}{i^{2} \rho_{i-1}}, 1\right) \\
& =t_{1}+t_{2}+t_{3} \text { say. }
\end{aligned}
$$

We proceed to estimate each of $t_{1}, t_{2}$ and $t_{3}$.

First we estimate $t_{1}$. If we use precisely the same argument which led to (44) then we would obtain

$$
\left|t_{1}\right| \leqslant 3 \text { if } j \text { were sufficiently large. }
$$

Next we estimate $t_{3}$. From (35) we know that $\rho_{j} \leqslant \rho_{j+1} \leqslant \rho_{j+2} \leqslant \ldots$, and $\lambda_{n j} \leqslant$ $\rho_{j}$. Therefore

$$
\left|t_{3}\right|=\left|H_{m, n_{j}}\left(\sum_{i=j+1}^{\infty} \frac{\psi_{i}(\cdot)}{i^{2} \rho_{i-1}}, 1\right)\right| \leqslant \lambda_{n_{j}}\left\|\sum_{i=j+1}^{\infty} \frac{\psi_{i}(\cdot)}{i^{2} \rho_{i-1}}\right\| \leqslant \frac{\lambda_{n_{j}}}{\rho_{j}} \sum_{i=1}^{\infty} i^{-2} \leqslant \frac{\pi^{2}}{6} .
$$

Finally we estimate $t_{2}$ which will turn out to be the dominant term in (46). Observe that, from Szabados [11, (7), (12)] it follows that, for $1 \leqslant k \leqslant n, \operatorname{sgn} A_{0 k n}(1)=(-1)^{k-1}$. Therefore, using Szabados [11, Corollary on p. 363] and letting $c$ denote various positive constants independent of $k, n$, we find that for $1 \leqslant k \leqslant n$,

$$
\begin{aligned}
(-1)^{k-1} A_{0 k n} & =\left|A_{0 k n}(1)\right| \\
& \geqslant c\left(\frac{\sqrt{1-x_{k}^{2}}}{n}\right)^{m} \cdot \frac{1}{\left(1-x_{k}\right)\left|x_{k}-x_{k \pm 1}\right|^{m-1}} \\
& \geqslant \frac{c}{n} \cdot \frac{\sin \theta_{k}}{1-x_{k}} \quad\left(x_{k}=\cos \theta_{k}\right) \\
& =\frac{c}{n} \cdot \sqrt{\frac{1+\cos \theta_{k}}{1-\cos \theta_{k}}} \\
& =c n^{-1} \sqrt{1+\cos \theta_{k}} .
\end{aligned}
$$

We now apply this observation to estimating $t_{2}$.

$$
\begin{aligned}
t_{2} & =H_{m, n_{j}}\left(\psi_{j}, 1\right) /\left(j^{2} \rho_{j-1}\right) \\
& =\sum_{k=1}^{n_{j}} \psi_{j}\left(x_{k, n_{j}}\right) A_{0 k n_{j}}(1) /\left(j^{2} \rho_{j-1}\right) \\
& =\sum_{k=k_{j}+1}^{K_{j}-1}(-1)^{k-1} A_{0 k n_{j}}(1) /\left(j^{2} \rho_{j-1}\right)
\end{aligned}
$$




$$
\begin{aligned}
& >\frac{c}{j^{2} \rho_{j-1}} \sum_{k=k_{j}+1}^{K_{j}-1} \frac{\sqrt{1+\cos \theta_{k}}}{k} \\
& >\frac{c}{j^{2} \rho_{j-1}} \sum_{k=k_{j}+1}^{K_{j}-1} k^{-1} \quad \text { for } j \text { sufficiently large } \\
& >\frac{c}{j^{2} \rho_{j-1}} \log \left(\frac{K_{j}}{k_{j}+1}\right)>\frac{c \log \log n_{j}}{j^{2} \log n_{j-1}}:=D_{j} \quad \text { say. }
\end{aligned}
$$

Now assume that the sequence $\left\{n_{1}, n_{2}, n_{3}, \ldots\right\}$ is diverging sufficiently quickly to ensure that $\lim _{j \rightarrow \infty} D_{j}=\infty$. Therefore we have

$$
t_{2} \geqslant D_{j} \text { and } \quad \lim _{j \rightarrow \infty} D_{j}=\infty
$$

If we apply the estimates (47), (49) and (48) to (46) we obtain

$$
\left|H_{m, n,}(\psi, 1)\right| \geqslant D_{j}-3-\pi^{2} / 6 \text { if } j \text { were sufficiently large. }
$$

Therefore $\limsup _{n \rightarrow \infty}\left|H_{m, n_{j}}(\psi, 1)\right|=+\infty$ and we have proved that equation (32) is satisfied. This completes the proof of Theorem 2 .

\section{REFERENCES}

[1] G.J. Byrne, T.M. Mills and S.J. Smith, 'The Lebesgue function for generalized Hermite-Fejér interpolation on the Chebyshev nodes', The ANZIAM J. 42 (2000), 98-109.

[2] L. Carleson, 'On convergence and growth of partial sums of Fourier series', Acta Math. 116 (1966), 135-157.

[3] R.A. DeVore and G.G. Lorentz, Constructive approximation (Springer-Verlag, Berlin, Heidelberg, New York, 1994).

[4] G. Grünwald, "Über Divergenzerscheinungen der Lagrangeschen Interpolationspolynome', Acta Sci. Math. (Szeged) 7 (1935), 207-221.

[5] G. Grünwald, 'Über Divergenzerscheinungen der Lagrangeschen Interpolationspolynome Stetiger Funktionen', Ann. Math. 37 (1936), 908-918.

[6] J. Marcinkiewicz, 'Sur la divergence des polynomes d'interpolation', Acta Litt. Sci. Szeged 8 (1936/1937), 131-135. (Also in A. Zygmund (ed.) Józef Marcinkiewicz: Collected Papers (Inst. Mat. Polskiej Akad. Nauk, Warsaw, 1964), pp. 204-209).

[7] I.P. Natanson, Constructive function theory III (Frederick Ungar, New York, 1965).

[8] T.J. Rivlin, Chebyshev polynomials: from approximation theory to algebra and number theory, 2nd ed. (Wiley, New York, 1990).

[9] S.J. Smith, 'On the fundamental polynomials for Hermite-Fejér interpolation of Lagrange type on the Chebyshev nodes', J. Inequal. Appl. 3 (1999), 267-277.

[10] S.J. Smith, 'Generalized Hermite-Fejér interpolation polynomials', Exposition. Math. (to appear). 
[11] J. Szabados," 'On the order of magnitude of fundamental polynomials of Hermite interpolation', Acta Math. Hung. 61 (1993), 357-368.

[12] J. Szabados and P. Vértesi, Interpolation of functions (World Sci. Press, Singapore, 1990).

[13] P. Vértesi, 'Practically $\rho$-normal pointsystems', Acta Math. Hungar. 67 (1995), 237-251.

Department of Mathematics

La Trobe University

PO Box 199

Bendigo Vic. 3552

Australia
Mathematical Institute of the

Hungarian Academy of Sciences

PO Box 127

Budapest H-1364

Hungary 